\title{
Strates
}

STRATES Matériaux pour la recherche en sciences sociales

13 | 2007

Paysage urbain: genèse, représentations, enjeux contemporains

\section{Montréal 1960, les ressorts d'une réidentification}

André Lortie

\section{(2) OpenEdition}

Journals

Édition électronique

URL : http://journals.openedition.org/strates/6083

DOI : $10.4000 /$ strates.6083

ISSN : 1777-5442

Éditeur

Laboratoire Ladyss

Édition imprimée

Date de publication : 31 décembre 2007

ISSN : 0768-8067

Référence électronique

André Lortie, « Montréal 1960, les ressorts d'une réidentification », Strates [En ligne], 13 | 2007, mis en ligne le 22 octobre 2008, consulté le 08 septembre 2020. URL : http://journals.openedition.org/ strates/6083 ; DOI : https://doi.org/10.4000/strates.6083

Ce document a été généré automatiquement le 8 septembre 2020

Tous droits réservés 


\title{
Montréal 1960, les ressorts d'une réidentification
}

\author{
André Lortie
}

On est allé la regarder du haut de la montagne et on a trouvé que c'était la plus belle. Et la plus calomniée [...]. Bord de l'eau, vieux Montréal ; bord des champs, le nouveau; bord de l'est, les Français ; bord de l'ouest, les Anglais; et le ciel audessus.

(F. Leclerc, Montréal, Paris d'Amérique $\left.{ }^{1}\right)$

1 En 1960, en préface d'un ouvrage consacré à Montréal, Paris d'Amérique, le poète Félix Leclerc oppose au couple vieille ville, ville nouvelle, celui des deux origines, du français et de l'anglais : à deux artefacts répondent deux langues, deux cultures. Ce biculturalisme se percevrait dans l'espace concret, dans une partition est-ouest de la ville, dont la frontière serait, dans la représentation mentale qu'ont les Montréalais de leur ville, la rue Saint-Laurent. L'image est simple, elle a la force de l'évidence. Elle suggère un espace social statique, celui des Deux Solitudes décrites par l'écrivain Hugh MacLennan en $1945^{2}$, ancré dans un espace concret non moins figé.

Les années 1960 inaugurent une nouvelle formulation de ce rapport. En 1969, à la fin de cette décennie, le romancier Mordecai Richler commente rétrospectivement la découverte de sa ville natale, à son retour de Londres :

Regagnant Montréal dix-neuf ans après, à l'été 1967 (l'été de notre glorieuse Expo), au retour par avion de Londres la démodée via New York la délabrée, je fus frappé par la prospérité de la ville. [...] J’y entrai par des autoroutes à voies superposées qui plongeaient ici, surgissaient là, débouchant sur un îlot de prospérité, un centre-ville hérissé d'immeubles de rapport et d'hôtels, ces derniers si neufs d'aspect qu'on les eût cru sortis de leurs caisses la nuit précédente. La Place Ville-Marie. Le métro. L'île Notre-Dame. Habitat [67]. La Place des Arts. Cette corne d'abondance ne pouvait être la ville où j'avais grandi et que j'avais désertée ${ }^{3}$. 


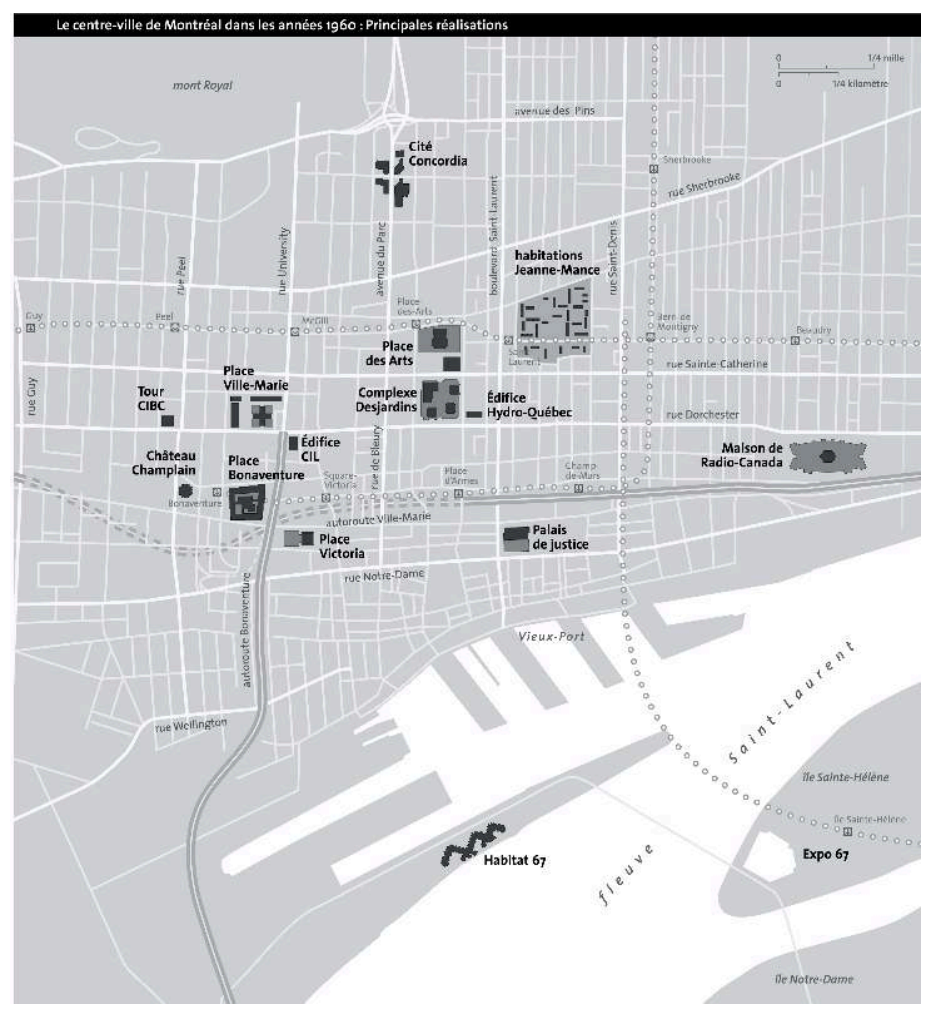

Fig. 1. Le centre-ville de Montréal dans les années 1960 : principales réalisations @ CCA, André Lortie, carte extraite de Les Années 60, Montréal voit grand, Montréal, Centre canadien d'architecture, Vancouver, Douglas \& McIntyre, 2004, p. 25. Cartographe : Eric Leinberger, 2004.

3 En une décennie à peine, la ville s'est métamorphosée. Sa morphologie, résultant de la rencontre entre une grille de rues orthogonales fondatrice et une topographie remarquable - mariage insulaire d'un mont et d'un fleuve -, est transcendée par un système de grandes voies rapides, de ponts, de viaducs, de tunnels, hérissé de gratteciel et d'immeubles-blocs. Dans cet espace devenu dynamique, le réseau du métro inauguré en 1966, qui franchit les frontières virtuelles sans formalités, se joue insidieusement des fondements de l'image collective de la ville décrits par Félix Leclerc, d'est en ouest et du nord au sud. Équipée de ces infrastructures, Montréal change d'échelle. Mais, à travers nombre de grands chantiers de rénovation et de développement, c'est son équilibre socioéconomique qui est aussi modifié.

4 Le géographe Guy Burgel, dans son ouvrage sur La ville, considère que cette dernière est la face spatiale de la sociétét. La ville en traduirait, dans l'espace, les spécificités. Il est vrai que le Québec change vite dans les années 1960, au rythme de la Révolution tranquille, et qu'il en est de même pour Montréal, qui en est le cœur économique et intellectuel. Quelle perception les Montréalais ont-ils de cette puissante métamorphose et comment s'identifient-ils à la ville moderne qui émerge sous leurs yeux ?

La dynamique, culminante en cette décennie, qui propulse le Québec de la campagne dans les villes, est alimentée par plusieurs phénomènes qui opèrent à des échelles différentes et qui contribuent à forger, aux yeux des Québécois, une image renouvelée d'eux-mêmes. Parmi les plus déterminants figurent: le facteur économique et ses ramifications mondiales; la politique nationale de planification et ses effets sur la transformation physique du territoire; l'effervescence politique, intellectuelle et 
culturelle propre à la minorité canadienne française ; et l'ouverture de cette minorité au monde.

6 Un des aspects de la mobilité économique concerne les apports de capitaux étrangers dans les grandes opérations immobilières qui, jusque-là, étaient restreintes aux opérateurs locaux. Les fonds sont sans commune mesure avec ce qu'ils étaient antérieurement, permettant des opérations d'une échelle nouvelle, tandis que, à l'inverse des investissements industriels, ce type de réalisation n'induit pas l'idée d'un rapport de subordination (société étrangère, main-d'œuvre locale), mais permet l'appropriation collective de l'objet de la valorisation qu'est l'immeuble. À l'échelle nationale, les avatars de la planification d'État touchent souvent les villes et contribuent à un double phénomène : un certain décloisonnement des enclaves locales par l'imposition de standards nationaux, dans le même temps que les modèles nationaux peuvent être appropriés localement et participer d'une identité spécifique, à l'instar, comme nous le verrons, de ce qui concerne le logement social.

7 Enfin, l'émancipation culturelle, intellectuelle et politique produit le meilleur et le pire : en même temps que se prépare l'Exposition universelle de Montréal de 1967, qui sera un formidable tremplin pour de nombreux artistes et intellectuels montréalais et québécois, les extrémistes du Front de libération du Québec montent en puissance, jusqu'aux événements tragiques qui verront leur dissolution en 1970. Pour les jeunes Canadiens français, l'accession aux pouvoirs politique et technique ainsi qu'à l'émancipation intellectuelle et culturelle, concomitantes d'une ouverture internationale dont l'Exposition universelle de 1967 est le point d'orgue, s'incarne en quelque sorte dans la transfiguration de leur métropole en pleine modernisation. D'autant que cette transformation profonde de la ville s'accompagne de la matérialisation de cette montée en puissance à travers de grands programmes institutionnels ou commerciaux propres à la portion francophone de la société québécoise.

8 Avant d'interpréter les signes de la réceptivité collective à cette transformation de l'espace métropolitain, qui participent d'un phénomène complexe de réidentification, il importe d'en résumer les grandes caractéristiques. Quelle est donc cette ville du premier $\mathrm{xx}^{\mathrm{e}}$ siècle, dont le Montréal des années 1960 se distinguerait tant ? Et quelles sont ces transformations majeures qui construisent ce renouvellement ${ }^{5}$ ?

La ville du premier $\mathrm{xx}^{\mathrm{e}}$ siècle

9 Au sortir de la Seconde Guerre mondiale, le manque de logements est estimé à 45000 , pour une population insulaire de l'ordre de 1,3 million d'habitants. L'insalubrité des quartiers populaires est décriée depuis nombre d'années, comme c'est le cas dans plusieurs métropoles occidentales par ailleurs. C'est notamment le Red Light District qui cristallise les représentations populaires de ce phénomène. Ce quartier de prostitution, qui prospère entre les deux guerres, accueillant une clientèle qui s'émancipe de la prohibition américaine, concentre les critiques dès lors que le Canada s'engage auprès de ses alliés et que les soldats de la fédération en permission y sont attirés. Rue de Bullion, « Rouged whores lean lips to narrow slits; they stop / The young soldier with his bag of salt ", écrit le poète Irving Layton à la fin des années $1940^{6}$.

10 Dans ce quartier de prostitution, déchéances physique et morale ne feraient qu'un. Habitat insalubre et populations nécessiteuses y seraient indéfectiblement liés. Les images qui en sont données éveillent les pires cauchemars des représentations populaires de la misère. Font écho à cet imaginaire des romans comme Le cassé, de 
Jacques Renaud, dont le héros se perd dans des rues et des ruelles aujourd'hui disparues ${ }^{7}$.

11 Ce secteur, dit du plan Dozois, est le premier visé, dans la décennie 1950, par les politiques de résorption de l'habitat insalubre et par celles de création de logements sociaux. Les habitations Jeanne-Mance, résultat de cette opération de rénovation urbaine, reçoivent leurs premiers locataires au tout début de l'année 1960. Cette réalisation est rendue possible par la convergence des actions municipale, provinciale et fédérale. Elle est le résultat d'une planification menée à l'échelle nationale par le biais de la Société centrale d'hypothèque et de logement ( $\mathrm{SCHL}$ ), dont l'agence d'architecture intégrée met au point des immeubles types en forme de barres, avec appartements mono-orientés et balcons. Les variantes de ces immeubles sont réalisées un peu partout au Canada, de Vancouver (Mclean Park et Skeena Street), à Halifax (Mulgrave Park), en passant par Montréal ${ }^{8}$. Pour autant, chaque municipalité développe sa propre réponse à cette normalisation des aides, ce qui témoigne, en ce qui concerne le logement collectif, d'une lente appropriation locale faite au détriment d'une politique de modèles. Le projet des habitations Jeanne-Mance restitue clairement les étapes de ce processus critique, puisque la douzaine d'immeubles types en hauteur conçus par l'architecte en chef de l'agence intégrée de la SCHL, Ian Maclennan, est en partie abandonnée pour des logements en bande sur trois niveaux.

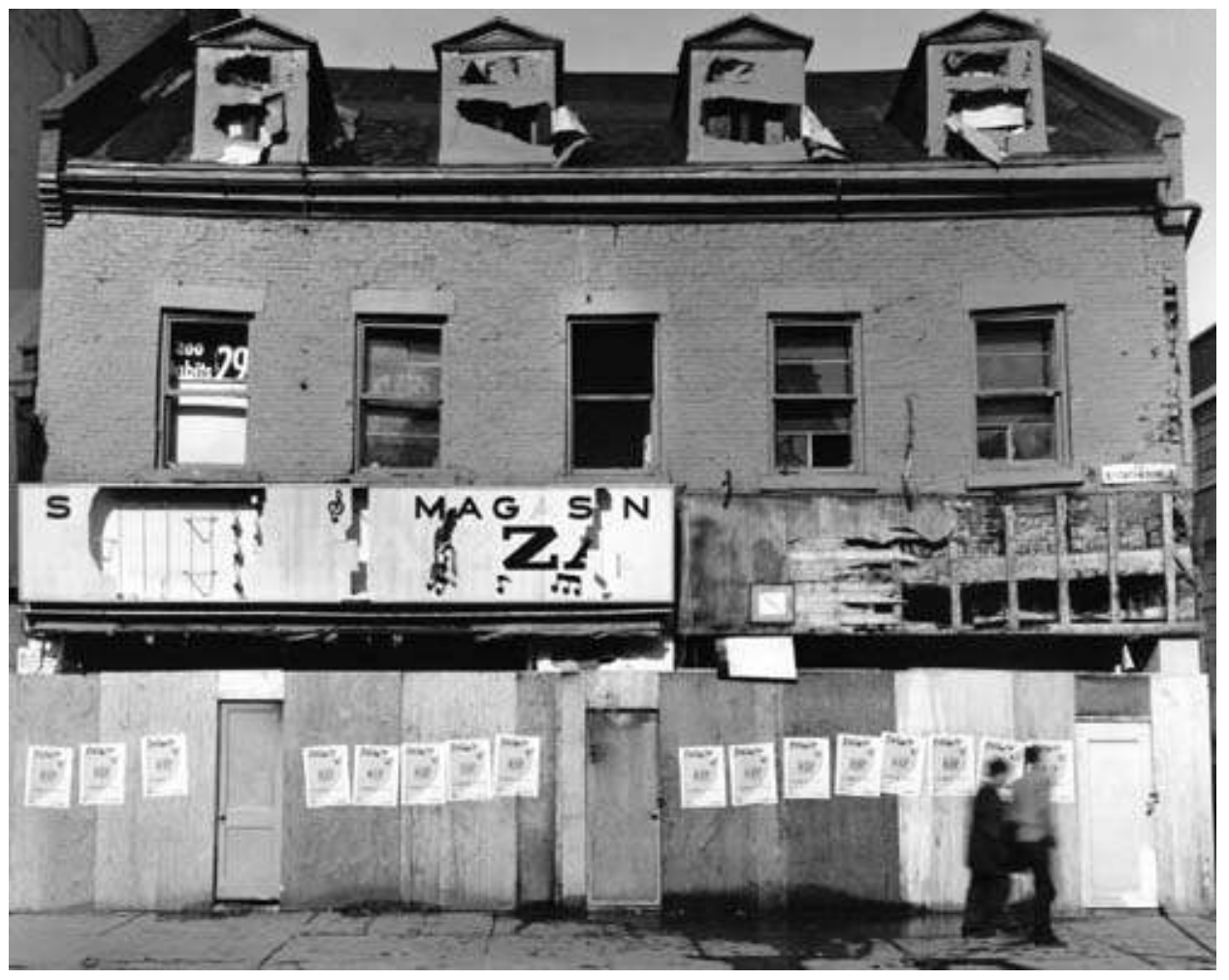

Fig 2. Montréal, un magasin de musique abandonné, 1963. 㯺 Jeremy Taylor.

12 Cette planification d'État ne concerne pas que le logement. Elle vise à résorber les disparités régionales au sein du territoire canadien développé. Elle touche la circulation des biens et des personnes (aéroports, route transcanadienne), la restructuration industrielle et, à la fin de la décennie, en préparation du centenaire de la confédération de 1867, l'équipement culturel. 
Une mutation structurelle

Les années qui suivent la Seconde Guerre mondiale voient émerger une nouvelle équation des transports et des déplacements à l'échelle continentale et internationale. Le bateau et le train, qui avaient fait la fortune de Montréal dans le système des échanges entre les centres urbains du Nord-Est américain, l'hinterland (matières premières et agriculture) et l'Europe, sont progressivement supplantés par le binôme avion-route. Si le grand chantier national du XIX $\mathrm{X}^{\mathrm{e}}$ siècle avait été celui de la voie de chemin de fer transcontinentale, c'est sans doute celui de l'autoroute transcanadienne qui, au $\mathrm{xx}^{\mathrm{e}}$, plus que le réseau de transport aérien, symbolise l'unification territoriale ${ }^{9}$. Elle n'est pas sans effets sur l'organisation urbaine. Tracée d'un océan à l'autre, l'autoroute transcanadienne traverse les grandes villes dans leur partie agglomérée, leur faisant subir des transits qui ne leur sont pas destinés. Ce phénomène est identique à celui que l'on observe à la même époque aux États-Unis, où des métropoles sont coupées d'autoroutes nationales en leur centre, comme Philadelphie par l'Interstate Highway $n^{\circ} 676$, une tranchée qui fend la ville pour mettre en communication le Delaware Bridge, à l'est, et la voie rapide sur la berge de la rivière Schuylkill, à l'ouest ${ }^{10}$, ou encore Boston, dont le centre historique est éventré par l'autoroute $n^{\circ} 93$.

De même qu'au XIx ${ }^{e}$ siècle, le chemin de fer était au service de l'industrie, les grandes infrastructures routières et autoroutières participent de la réorganisation de l'appareil de production. Le train et le bateau avaient eu tendance à concentrer les échanges à proximité du centre: il est évident que la grande route, et surtout l'avion, s'en dissocient, et que l'industrie se délocalise en conséquence. La désindustrialisation des centres urbains canadiens ne fait pourtant pas l'objet d'une politique concertée. Elle est plutôt l'effet induit d'une action gouvernementale qui accompagne un mouvement de concentration industrielle, prolongeant les restructurations exigées par l'effort de guerre $^{11}$. Ce mouvement a tendance à absorber l'artisanat, le commerce, la petite ou moyenne industrie familiale. Ceux-ci s'accommodaient d'une situation proche des centres et d'une main-d'œuvre sur place. L'encouragement à la consolidation industrielle amène un changement d'échelle qui induit son déplacement le long des nouvelles grandes infrastructures, hors des centres, loin du logement ouvrier traditionnel. Elle laisse le cœur des villes dévitalisé, orphelin de ses sources d'emploi, désarticulant un tissu urbain dans lequel les ouvriers vivaient souvent à proximité de leur lieu de travail, à distance de tramway, comme Jules Lebœuf, héros du roman de Gérard Bessette, La bagarre ${ }^{12}$.

15 Cette dynamique induit une perte de valeur au centre, qui rend plausible le projet de dissociation fonctionnelle réclamée depuis des décennies par les réformateurs sociaux et hygiénistes, auquel les politiques fédérales d'aide au logement apportent leur appui. Parallèlement, la concentration tertiaire au cœur de la ville, dans des immeubles spécialisés, poursuit une tendance déjà engagée depuis la fin du siècle précédent. Elle atteint toutefois un niveau inattendu à Montréal, avec la construction, au tournant des décennies 50 et 60 , de $150000 \mathrm{~m}^{2}$ de bureaux à la Place Ville-Marie - sur laquelle nous reviendrons -, une opération qui stimule l'imagination des promoteurs locaux et des investisseurs internationaux qui lui emboîtent le pas. Ce mouvement de concentration immobilière dans des programmes complexes consolidant un ou plusieurs îlots urbains n'est pas neuf en Amérique du Nord. Il suffit d'évoquer le Rockefeller Centre de New York pour s'en convaincre. Il est toutefois inédit à Montréal et hisse la ville au 
niveau de tels grands centres, bientôt imitée ailleurs au Canada, à Toronto notamment ${ }^{13}$, faisant rêver les investisseurs jusqu'à Vancouver ${ }^{14}$.

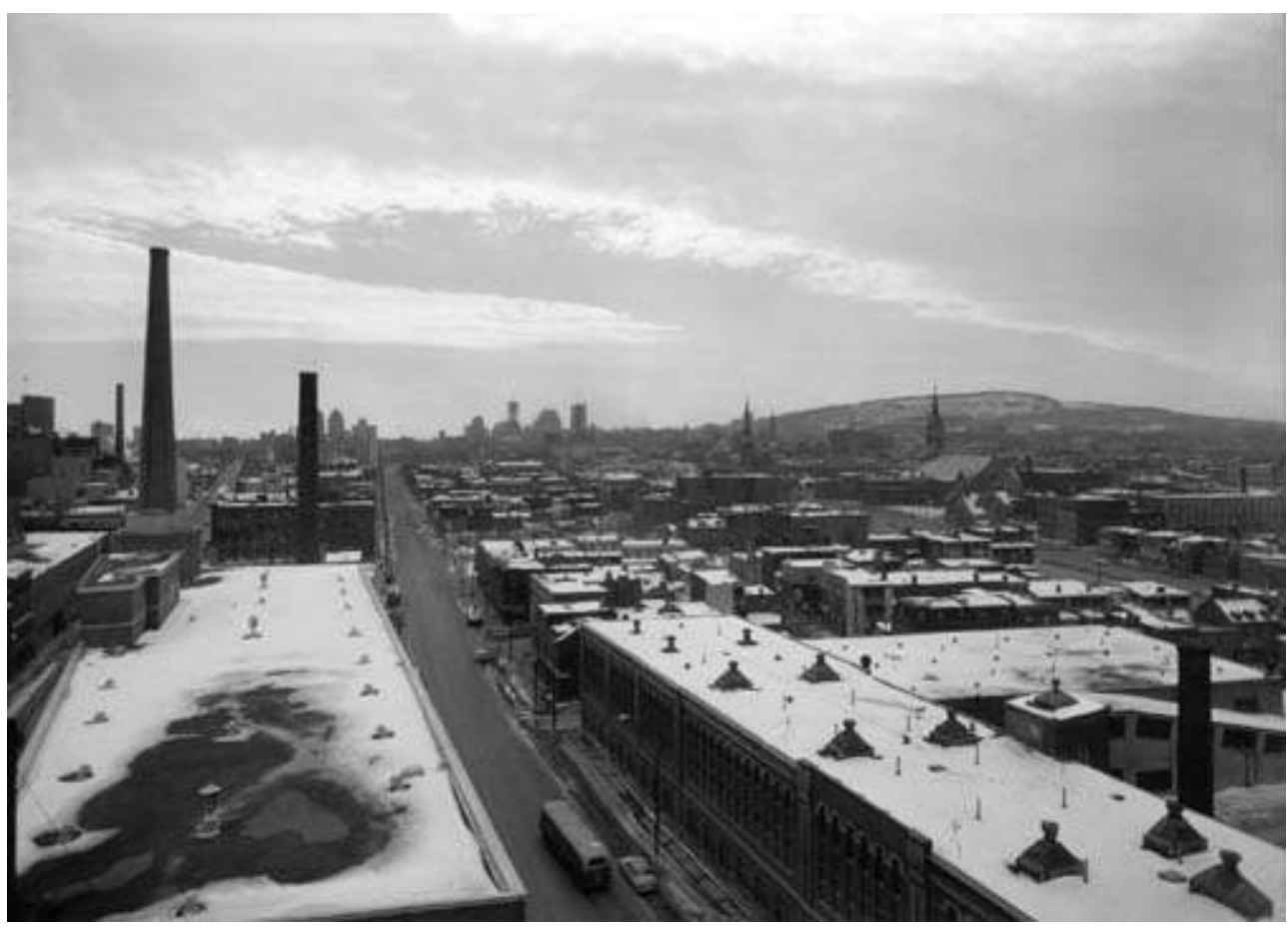

Fig. 3. Montréal, cheminées et clochers vus depuis le pont Jacques-Cartier, 1961. 鹥 Jeremy Taylor.

Dans le même temps, la SCHL fait la promotion de la maison individuelle, pour laquelle elle produit aussi des modèles d'unité et de lotissement. En effet, si l'industrie quitte les centres, encore faut-il lui offrir à proximité la main-d'œuvre qui lui est nécessaire. Avec le discours de son président prononcé devant la Royal Commission on Canada's Economic Prospects en 1956, on retrouve des accents planistes plus familiers d'un pays comme la France :

De par sa place fondamentale dans une économie industrielle moderne et ses profondes implications sociales, le logement est une question qui interpelle inéluctablement et profondément tous les paliers de gouvernement. La fourniture de logements s'apparente, par certains aspects, à un service public ${ }^{15}$.

17 La planification nationale se noue clairement autour de l'enjeu du logement, qui associe grande industrie, infrastructures de transport et organisation des communautés sur le territoire :

La création de zones résidentielles suppose la conception de réseaux de rues et de services publics ainsi que la localisation de centres commerciaux, d'écoles et de lieux du culte. La construction domiciliaire constitue donc la première étape de la modélisation des configurations de collectivité qui influeront sur l'existence de nombreuses générations ${ }^{16}$.

Changement d'échelle

Dès lors, l'urbanisation montréalaise se déverse sur l'île et au-delà, vers l'île Jésus, au nord, ou sur la rive sud du Saint-Laurent. Le bien nommé boulevard Métropolitain, ouvert à la circulation en 1960, et qui traverse l'île de Montréal suivant son grand axe, est à cette image, avec son chapelet d'industries, de lotissements de maisons individuelles, de petits complexes tertiaires, de centres commerciaux... 
Encouragé par la SCHL, les maisons individuelles et autres bungalows prospèrent au bout des nouvelles infrastructures, comme par exemple sur la rive sud du fleuve, de l'autre côté du pont Champlain inauguré en 1962. C'est le cas de la ville de Candiac, créée à partir de rien: "Candiac, la ville moderne où il fait bon vivre ${ }^{17}$." Ville de pavillons produite par la promotion immobilière privée, isolée de tout et dans laquelle on a oublié les commerces et les équipements publics...

Ce redéploiement métropolitain ouvre le champ à une dynamique de renouvellement urbain sans précédent au cœur de Montréal. La mobilité internationale grandissante des capitaux alimente un changement d'échelle au centre de la ville. Ce cycle est inauguré par le projet de Place Ville-Marie, que le promoteur immobilier visionnaire William Zeckendorf, venu de New York, lance dès le milieu des années 1950. Place VilleMarie, qui donne naissance à un axe de valorisation à l'ouest, avec les projets à venir de place Bonaventure (premier méga-bloc montréalais, traversé par les voies de chemin de fer de la gare Centrale, combinant une immense halle d'exposition, des bureaux, un hôtel de 400 chambres et trois restaurants, un jardin à ciel ouvert, des galeries commerciales, des aires de livraisons, un accès au métro, le tout posé sur la voie rapide est-ouest Ville-Marie), place Victoria (complexe boursier réalisé par l'Immobiliare di Roma, par l'architecte Luigi Moretti) ou du Château Champlain. On va jusqu'à imaginer des conquêtes colossales, comme celle des voies ferrées de la gare centrale, que les architectes André Blouin et Victor Prus ${ }^{18}$ imaginent couvrir d'une dalle de plusieurs hectares, lancée jusqu'aux rives du canal de Lachine et des bassins du port industriel.

Si Place Ville-Marie est le premier jalon d'un cycle spontané d'intense valorisation foncière, en revanche, à l'est, c'est par une décision technique et politique que la société canadienne française se donne les moyens de manifester, dans l'espace de la ville, sa montée en puissance, à la fois culturelle et économique. Place des Arts (deux auditorium dont un de 3000 sièges), le siège de l'Hydro Québec (société issue de la nationalisation de l'industrie électrique québécoise), le complexe Desjardins (trois tours de bureaux, un hôtel de voyageurs et une galerie commerciale dont l'étude commence en 1966), l'Institut de technologie de Montréal (non réalisé), la place de la Confédération (conçue autour d'un stade de 80000 places, non réalisée), Radio Canada (réalisé plus à l'est), sont autant de projets qui matérialisent cet axe nord-sud de l'est, réponse socioculturelle canadienne française à la valorisation capitalistique de l'ouest, attribuée à la fraction anglophone de la société montréalaise. 


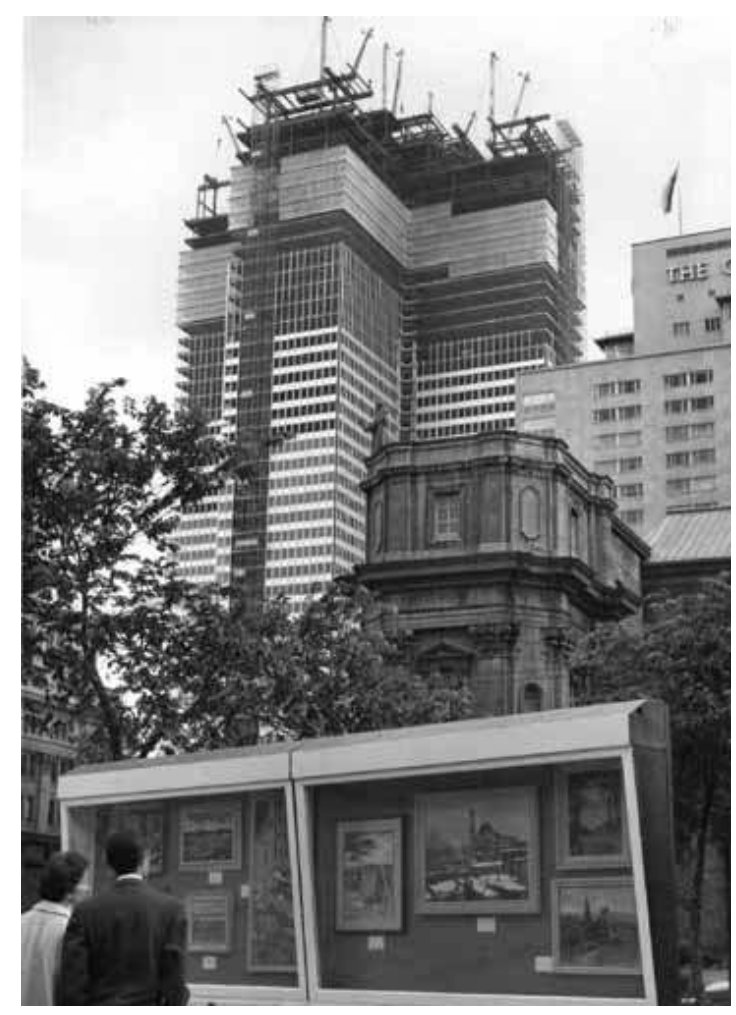

Fig. 4. Montréal, une exposition de tableaux en plein air, avec le chantier de Place Ville-Marie en arrière-plan, 1961. 圈 Jeremy Taylor.

Il serait toutefois inexact d'imaginer que ces phénomènes puissent être spontanés et délaissés de toute volonté politique. Deux facteurs essentiels agissent à l'époque comme catalyseur et accélérateur. Il y a d'abord les prévisions démographiques qui estiment que la population de la région de Montréal devrait atteindre 4,8 millions en 1980 et plus de 7 millions en l'an 2000, alors qu'elle est de 2,4 millions en 1960 et atteint environ 3,3 millions aujourd'hui. Il y a ensuite l'Exposition universelle de 1967 qui met tous les gouvernements d'accord pour réaliser les infrastructures prévues dans le cadre du grand projet de transformation pensé pour ce Montréal de 5 millions d'habitants: ponts, voies rapides, autoroutes, tunnels, etc.

Ainsi, alors que la ville contemporaine s'invente au cœur de Montréal, avec des tours, un métro, des galeries commerciales souterraines, des autoroutes urbaines, des immeubles-blocs, etc., au milieu du fleuve, en position de miroir face à ce centre en pleine métamorphose, l'Exposition universelle de 1967 est l'occasion de mettre en chantier un laboratoire de projets parmi les plus en pointe de l'architecture et de l'urbanisme: mégastructures, préfabrication, modularité, transparence et contrôle climatique, transports collectifs à vitesses coordonnées...

Montréal allégorie de la modernité

Dans un numéro de 1966 de la revue américaine Architectural Forum, c'est sous la plume de son directeur de la rédaction, Peter Blake, que l'expérience montréalaise est accueillie comme un accomplissement et une promesse. La métropole canadienne semble devoir réaliser les pensées les plus engagées de l'architecture contemporaine :

Through a happy combination of expert foresight, private initiative, and luck, Montreal is about to become the first twentieth-century city in North America [...] What sets this core apart is not so much the towers as their spreading roots in a 
multilevel network of shops, transportation systems, and pedestrian promenades

(P. Blake, « Downtown in 3D ») ${ }^{19}$.

Friedman, Kenzo Tange ou Alison et Peter Smithson, Montréal devient une caisse de résonance pour divers projets utopiques ou manifestes, tels ceux de Moshe Safdie pour Cité des îles, qui poursuit ses réflexions issues de Habitat 67, ou Zvi Hecker, qui imagine une ville mégastructurelle suspendue au-dessus du fleuve Saint-Laurent, ou encore Peter Cook, du groupe Archigram, avec sa Tour pour Montréal. Des projets qui prolongent, en quelque sorte, la modernité montréalaise en une prospective théorique, localisée et réactive.

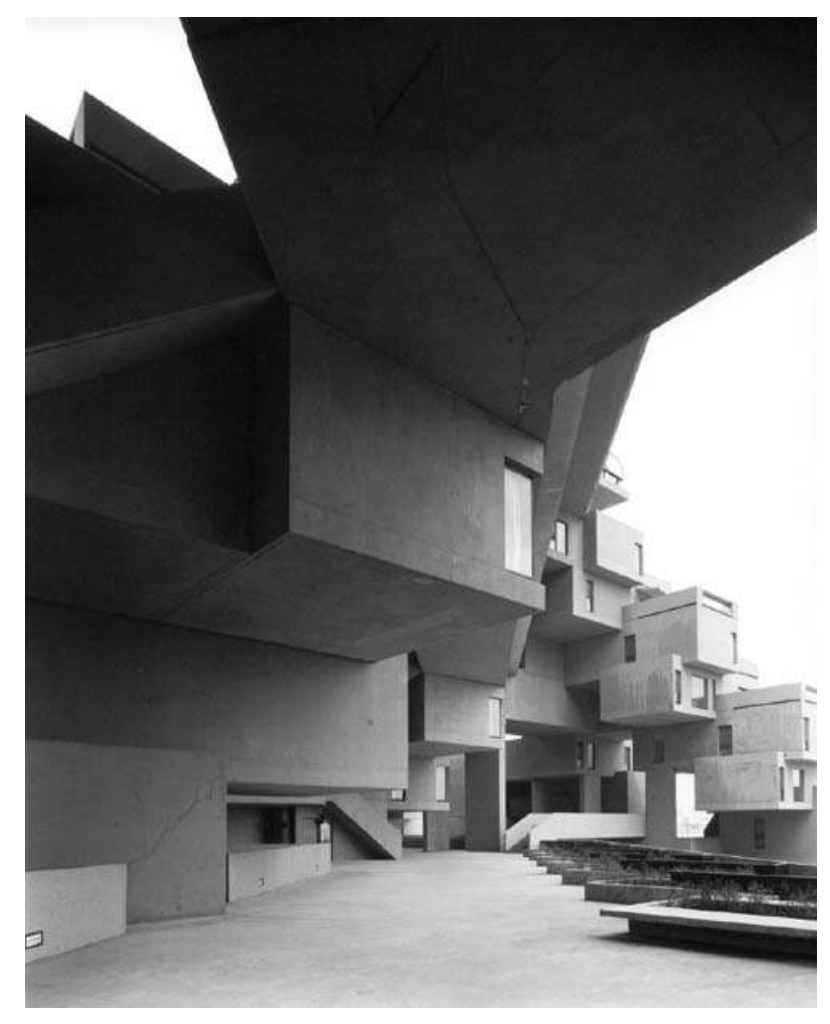

Fig. 5. Montréal, une coursive extérieure, Habitat 67, Moshe Safdie, architecte, 1967. 眑 Jeremy Taylor.

Pourtant, à l'Exposition universelle de 1967, dans la parade de styles qui s'offre au public de toutes les nations, un pavillon attire l'attention du monde de l'architecture (celle de Peter Blake, à nouveau, mais aussi de Sybil Moholy-Nagy). Il s'agit du pavillon du Québec. La critique du New York Times y voit le nouveau « pavillon de Barcelone », en référence à l'œuvre de Mies van der Rohe édifiée pour l'Exposition universelle de 1929. Or, il s'agit de l'une des réalisations parmi les plus dépouillées de l'exposition. En mur rideau de verre miroir suspendu, il est à l'image de la ville qui se construit en face : sophistications technique et programmatique, alliées à une certaine sobriété 
architecturale. Et le message qu'il délivre dans son contenu est celui d'un engagement sans faille dans la modernité.

Cette facture résolument moderne est en phase avec le message de vitalité canadienne française qui est au cœur du projet pour ce pavillon, qui fait les louanges d'un peuple qui a su maîtriser les éléments afin de construire son identité dans un territoire hostile. Il est, en quelque sorte, le point d'orgue d'une réidentification des Québécois à leur propre environnement qui n'est plus celui des champs et des bois, mais celui de la ville, une ville en train de glisser de l'industrie vers le tertiaire.

Déjà, dans L'invention de la mort ${ }^{21}$, manuscrit des années 1950 publié après son décès, Hubert Aquin faisait le choix d'installer son héraut au-dessus de l'animation urbaine, dans une chambre de l'hôtel Queen Elisabeth juste construit. C'est depuis cet observatoire offert par la ville moderne qu'il prenait ses distances par rapport au monde dont il se détachait lentement. Quelques années plus tard, c'est au sommet du même hôtel que Jean Cimon observe :

[...] dans l'azur matinal, les gratte-ciel tout blancs prennent un bain de soleil! [...] Triomphe du Montréal vertical dont les jeunes tours encore clairsemées s'envolent joyeusement dans le ciel. Hélas! il faut redescendre dans la rue Dorchester et constater le manque de vision de nos « élargisseurs » de rues. L'occasion était belle de créer un ruban de verdure à travers le cœur de la ville, une sorte de Central Park linéaire qui aurait enchanté le piéton et permis d'admirer à loisir la fulgurante beauté des gratte-ciel de l'avenir'22!

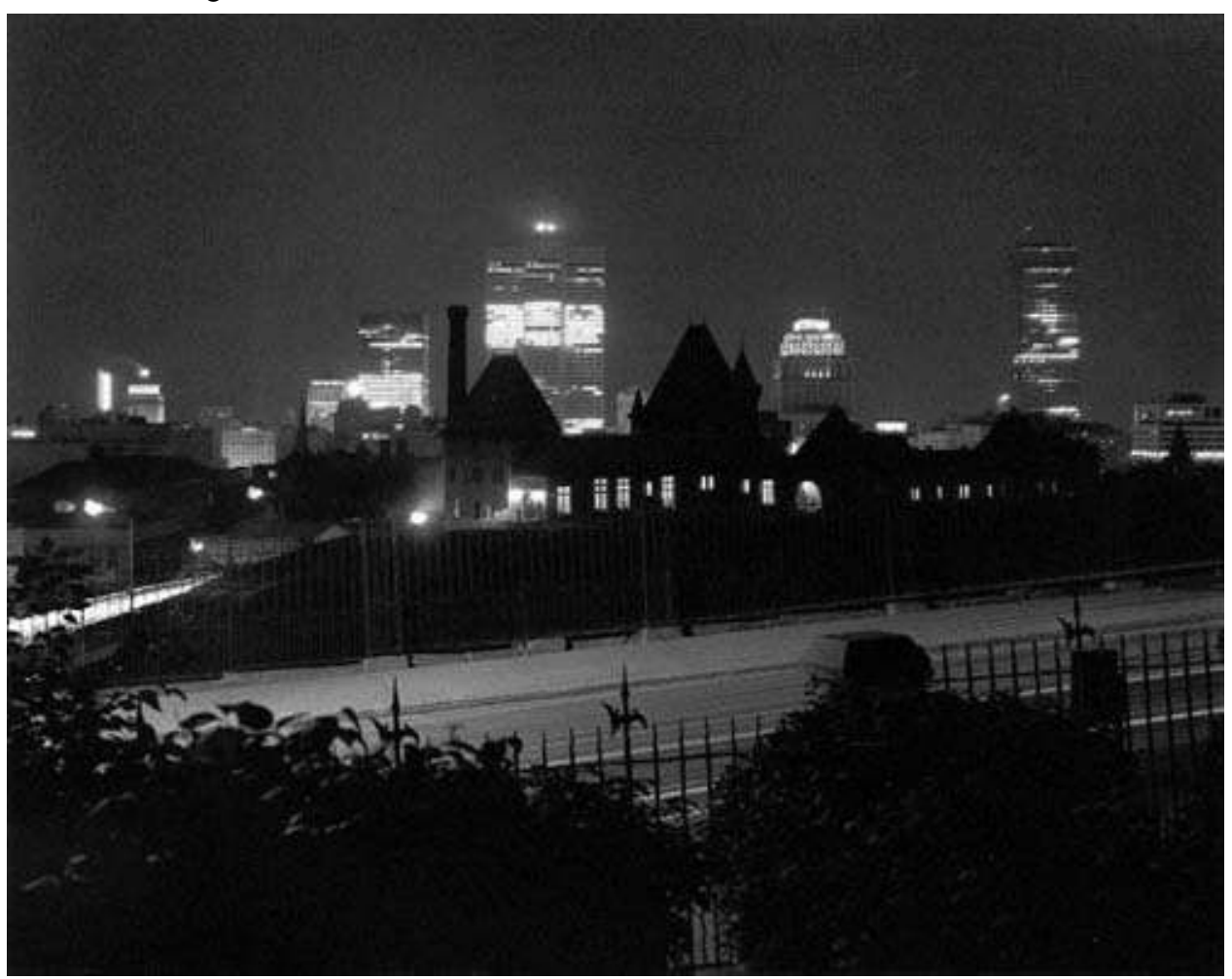

Fig. 6. Montréal la nuit, 1963. 瞇 Jeremy Taylor.

Mais c'est peut-être dans les images filmées par le cinéaste Gilles Carle que la fascination pour la ville moderne affleure avec plus de sensibilité, lorsque l'immeuble de la Place Ville-Marie (encore lui) surgit dans La vie tranquille de Léopold $Z$, illuminé dans la nuit d'hiver et les flocons de neige, au détour de rues étroites. Cette solution de 
continuité est à l'image de celle qui apparaît dans les guides touristiques, qui passent d'un quartier à l'autre, d'une ville à l'autre, moderne, historique, ouvrière ou universitaire, en tournant une page, en plongeant dans un chapitre par l'intermédiaire d'un index. Une solution de continuité qu'offre à sa manière le métro, dont les couloirs débouchent au cœur même de ces icônes de la modernité : complexe de la Bourse (Moretti, arch. et Nervi, ing.), Westmount Square (Mies van der Rohe, arch.), place Ville-Marie (Ieoh Ming Pei et Henry N.Cobb, arch.)... Loin du désert vilipendé par certains critiques des années 1970, ce dernier complexe est plébiscité par l'American Girl-Watching Association, dans son classement mondial des sites les plus propices à l'observation des spécimens de la gente féminine ${ }^{23}$.

31 Comme on peut le constater lors du défilé de la Saint-Jean-Baptiste de 1965, Montréal ne figure-t-il pas, aux yeux des Montréalais eux-mêmes, l'allégorie de la modernité : "Montréal ville historique et moderne » proclame l'inscription d'un char allégorique, dont les gratte-ciel stylisés, entrecoupés de maisons en rangées traditionnelles et d'arbres, au pied desquels la jeunesse montréalaise défile assise, évoquent l'immeuble de la CIL, dessiné par les architectes new-yorkais de renommée internationale Skidmore, Owings et Merrill, ou les tours jumelles de la Bourse, square Victoria, alors à l'étude.

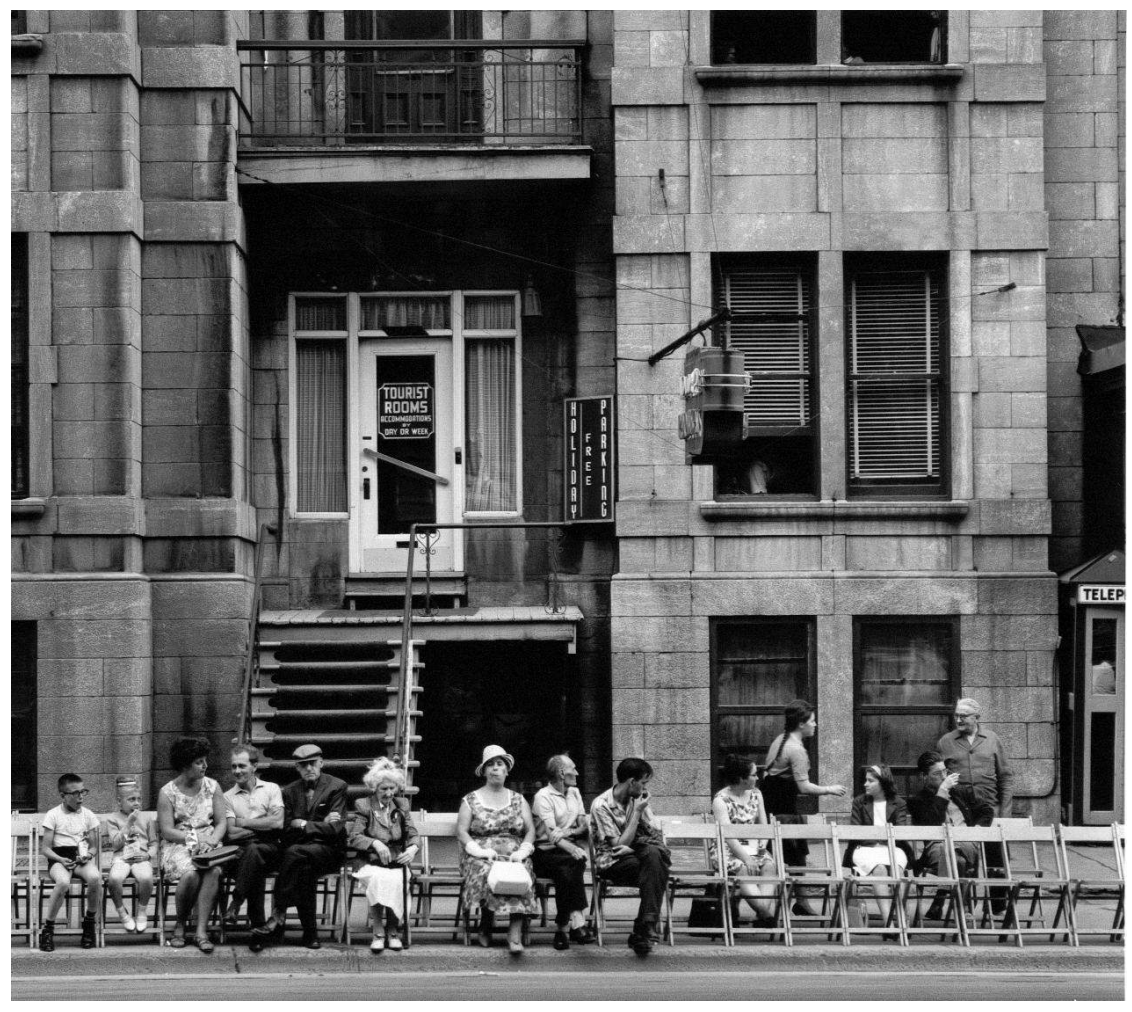

Fig. 7. Montréal, l'attente du défilé de la Saint-Jean Baptiste, 1964. 围 Jeremy Taylor.

Pourtant, la rupture d'échelle qu'impose cette ville moderne n'est pas sans poser quelques questions aux mêmes Montréalais. Pierre Gravel, dans À perte de temps s'inquiète :

À vrai dire, la ville n'existait pas. À sa place, une chose grosse, énorme, monstrueuse, qui n'avait pas été nommée, se déployait autour d'une montagne et se terminait en s'amenuisant en banlieues: l'est, l'ouest, le nord, quelques taudis 
dispersés... Au centre, le verre et les poutres [...] On pouvait difficilement, dans ces conditions, parler d'une ville. Et effectivement, on n'en parlait pas : on y vivait ${ }^{24}$.

Hubert Aquin, dans son « essai crucimorphe » sur la symbolique de la Place VilleMarie, cette dernière résume «l'ambiguïté ontologique du Montréal moderne: [une] ville [qui] n'est pas tout à fait à l'image de ceux qui l'habitent, ni même un reflet de ceux qui la possède ». «À l'image de Montréal dont elle est le cœur artificiel, la Place Ville-Marie est un agent double, [...] enfant naturel de notre biculturalisme ${ }^{25}$. »

Il est vrai que ce glissement vers la modernité se fait par effacement de pans entiers de la ville ouvrière, de ses populations délocalisées et de ses usages. Ainsi, dans le même temps que les Canadiens français s'identifient à cette ville moderne qui émerge sous leurs yeux, ils (re)découvrent la ville issue de l'histoire que cette modernisation met en péril.

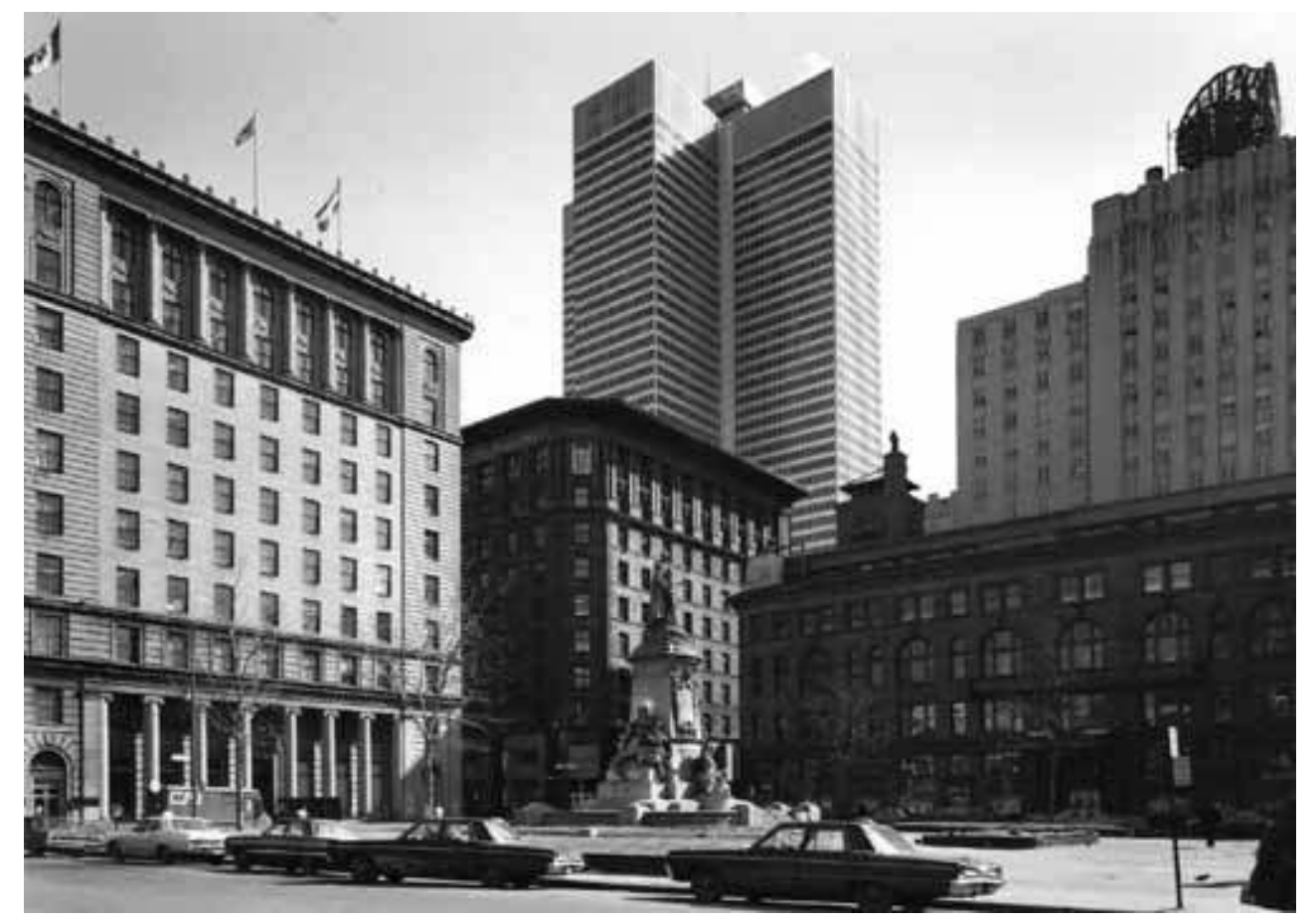

Fig. 8. Montréal, le square Dominion, avec l'immeuble de la Place Ville-Marie en arrière-plan, 1967. 匑 Jeremy Taylor, DR.

\section{Identité et réidentification}

Et la disparition de la ville issue de l'histoire ne laisse pas les Montréalais indifférents. Photographes et écrivains s'en inquiètent, de même que les habitants des quartiers visés par certaines rénovations. Le photographe Michel Saint-Jean en fait un relevé systématique dans le Faubourg à la Mélasse, avant les destructions qui précèdent la réalisation de Radio-Canada, comme le fera Brian Merrett quelques années plus tard à Milton Parc, le quartier populaire mitoyen de la grande université anglophone montréalaise, McGill University, nommé d'après les rues qui se croisent en son centre et menacé de destruction par la démesure des projets de la promotion immobilière. 


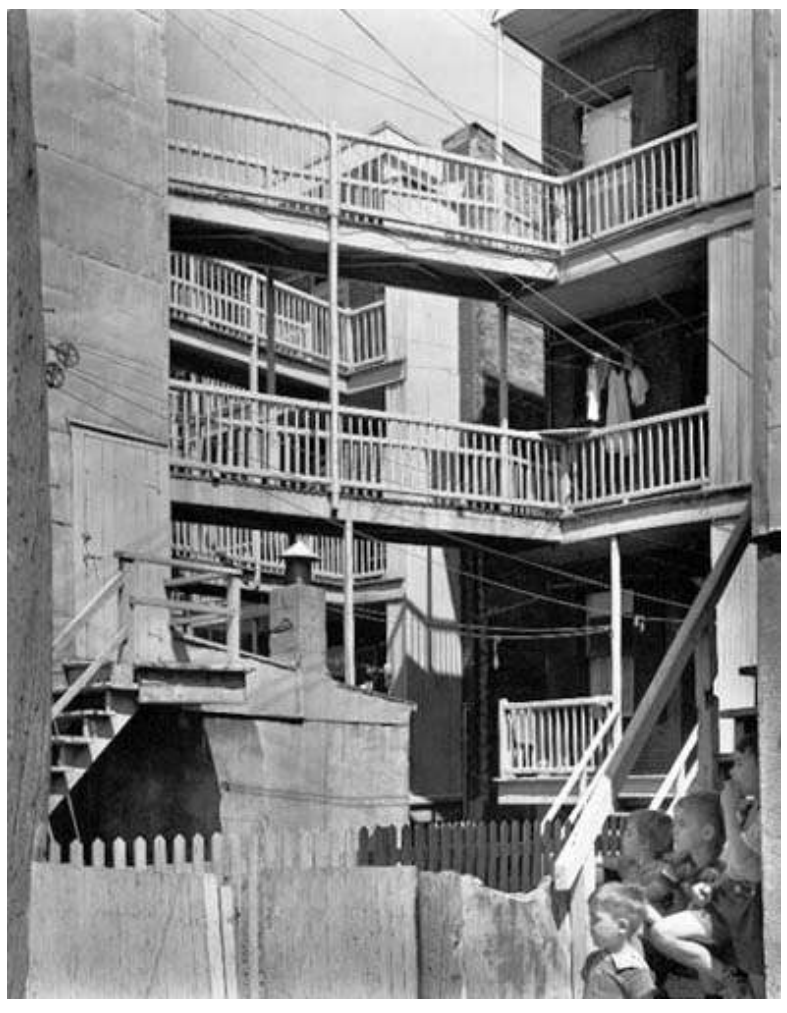

Fig. 9. Montréal, jeunes garçons au balcon, 1963. 鹋 Jeremy Taylor, DR.

C'est d'ailleurs l'écrivain anglophone Hugh Hood qui fait des descriptions enthousiastes des rues populaires francophones dans son recueil de nouvelles Around the Mountain, Scenes from Montreal Life, publié l'année de l'Expo:

Ah, but Henri-Julien, Drolet, that's the real thing... They are narrow blocks, buildings fronting on parallel main street and backing on a shared alley. Each long block is made vertebrate by the crawling teeming ruelle life... Sometimes back there you'll find a small, balding, grass plot with ten adults roosting on it and God knows how many kids hollering ${ }^{26}$.

37 Sans doute Jeremy Taylor, auteur des images qui illustrent cet article, est-il l'un des photographes qui a le mieux cerné l'ambiguïté et l'ambivalence des forces contradictoires qui façonnent alors le visage de Montréal. Ses prises de vue traduisent cette fascination pour une modernité à laquelle les Montréalais adhèrent, malgré les ruptures dont ces artefacts sont porteurs, tout en restant attachés aux formes urbaines traditionnelles, débarrassées toutefois de leur signification historique de classe. 


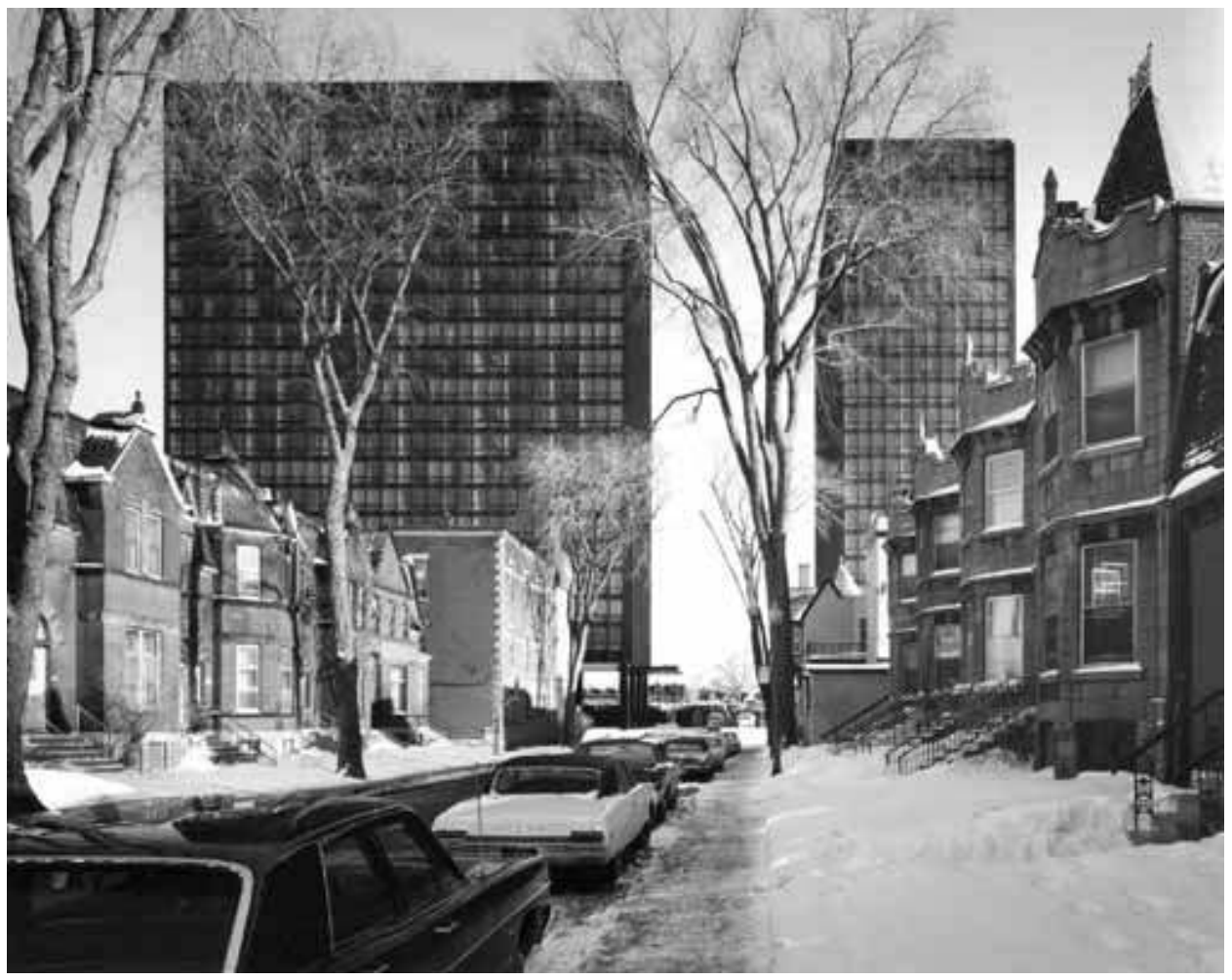

Fig. 10. Montréal, le Westmount Square (Mies van der Rohe, architecte), vu depuis la rue Elm, 1968. 弱 Jeremy Taylor, DR.

38 La brutalité de certaines rénovations n'est toutefois pas sans attiser les luttes urbaines, dont la chronique est commentée jusqu'en Europe ${ }^{27}$. Elles visent des populations qui ne se sentent pas moins légitimes à vivre dans ces quartiers du centre dont ils se voient progressivement exclus. Roch Carrier fait la description de cette violence dans son ouvrage de 1973, Le deux millième étage :

Dorval n'arrivait pas à croire que cette destruction avait lieu dans son quartier, ce lieu où il avait toujours habité, depuis qu'il avait choisi d'habiter quelque part. L'on démolissait les maisons des seules personnes qu'il connaissait au monde. Le bulldozer attendait de foncer dans sa maison. L'on jetait en poussière, en cassures de briques, plus de vingt années vécues par lui ${ }^{28}$. 


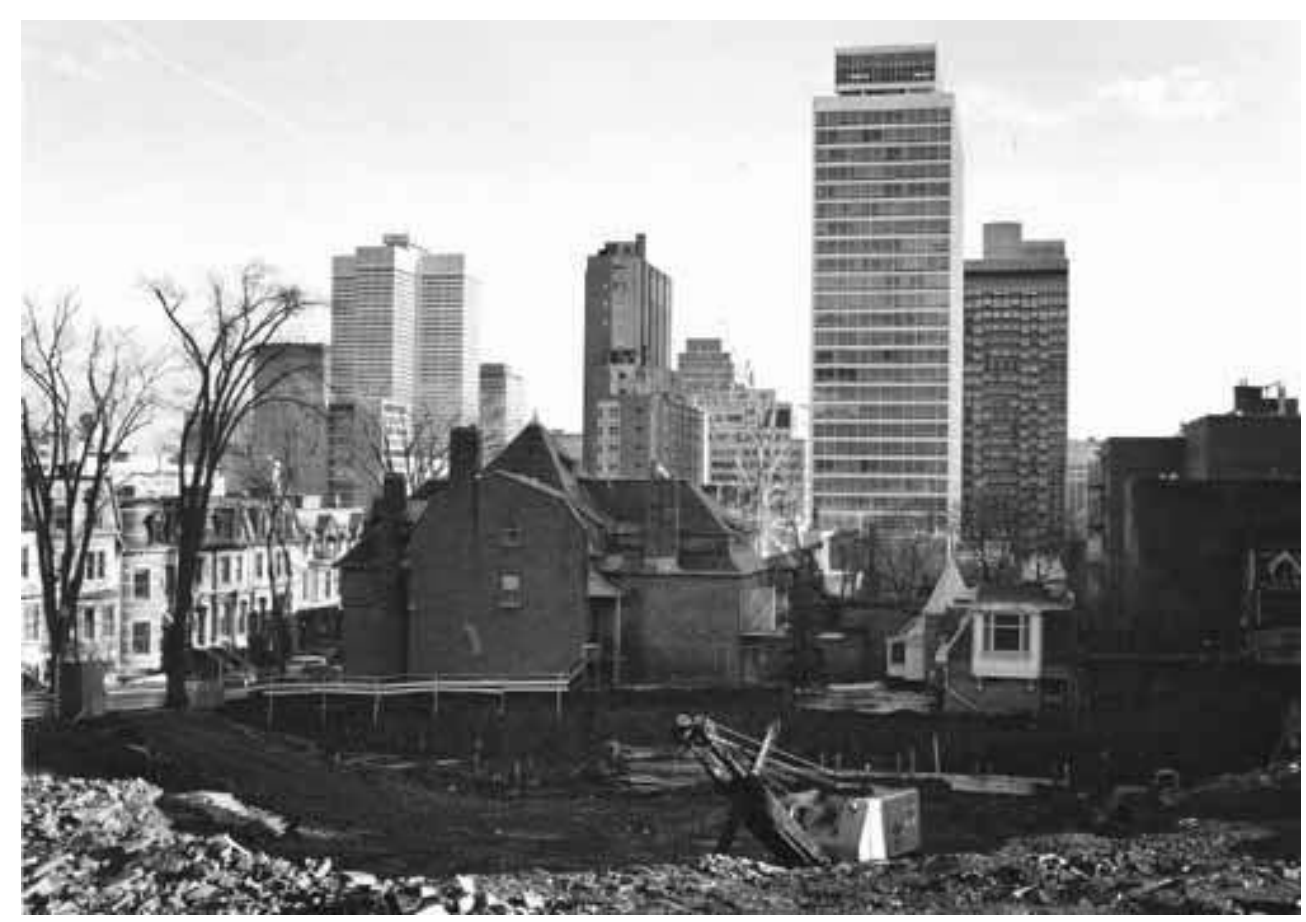

Fig. 11. Montréal, démolitions et excavations préalables à la construction d'une opération de logements, 1967. 䱢 Jeremy Taylor, DR.

1973, année de la démolition de la maison Van Horne, est un véritable tournant. Construite au XIX ${ }^{e}$ siècle, au cœur du «mille carrée ${ }^{29}$ ", ce flanc sud du mont Royal occupé par les opulentes résidences de la bourgeoisie capitalistique anglophone, la maison Van Horne, du nom de son propriétaire, le président de la compagnie des chemins de fer Canadien Pacifique, se trouvait aux franges de la partie du centre ville de Montréal en pleine mutation. Alors que son récent propriétaire souhaitait la démolir afin de réaliser un immeuble de grande hauteur, les associations de défense du patrimoine tentaient d'obtenir son classement. Vaine démarche, puisque le 10 septembre 1973, la maison tombait sous le pic des démolisseurs. Certes, l'événement devait provoquer une certaine prise de conscience quant à la fragilité des intérêts culturels face aux appétits financiers. Mais c'était aussi l'indice que non seulement les Montréalais prenaient la mesure de l'intérêt de leur patrimoine urbain, mais que francophones et anglophones pouvaient faire cause commune pour sauver une demeure du XIX ${ }^{e}$ siècle, symbole d'asservissement pour les uns et de domination pour les autres.

C'est le début d'une nouvelle ère. Elle clôt une période de prospérité et de violence, qui aura vu l'explosion des luttes armées du Front de libération du Québec cohabiter avec celle des gratte-ciel et des galeries commerciales. "Maître chez nous ", lançait, en guise de projet politique, le Premier ministre du Québec, Jean Lesage, élu en 1960. Rétrospectivement, l'éradication de pans entiers de la ville ouvrière semble avoir été le prix de la réconciliation des Canadiens français avec leur propre histoire, le stimulateur d'une prise de conscience des richesses et des ressources de leur propre culture. Dans le même temps, les Montréalais s'identifiaient fortement à cette ville moderne, souterraine et aérienne, qui s'érigeait sous leurs yeux. Une double condition nécessaire pour se concevoir comme partie d'une histoire internationale. 


\section{NOTES}

1. F. Leclerc, «Préface », in M. Régnier, Montréal, Paris d'Amérique, Paris of America, Ottawa, Éditions du Jour, 1961.

2. H. MacLennan, Two Solitudes, Montréal, McGill-Queen's University Press, 2006 (1re édition en 1945).

3. M. Richler, The Street, Toronto, McClelland \& Stewart, 1969, p. 5.

4. G. Burgel, La ville aujourd'hui, Paris, Hachette, 1993, p. 133.

5. Cet article est issu d'un projet de recherche, d'exposition et de publication mené par le Centre canadien d'architecture. A. Lortie (dir.), Les années 60, Montréal voit grand, Montréal, Vancouver, CCA, Douglas \& McIntyre, 2004, 216 p.

6. Les prostituées fardées susurrent avec insistance à travers d'étroites fentes / elles attirent le jeune soldat et son sac de sel (traduction de l'auteur). I. Layton, « Rue de Bullion ", in A Red Carpet for the Sun, 1959.

7. J. Renaud, Le cassé, Montréal, Parti Pris, 1964.

8. Voir les numéraux spéciaux du Journal de l'Institut royal d'architecture du Canada, vol. XXXVI, janvier 1959, p. 21 ; vol. XXXVII, août 1960, p. 330.

9. L. Mc Nally, « Roads, Streets, and Highways ", in N. R. Ball (dir.), Building Canada, a History of Public Works, Toronto, University of Toronto Press, 1988, p. 51. À titre d'exemple, voir la conséquente filmographie des années 1950 et 1960 consacrée par l'office national du film du Canada à ce grand œuvre.

10. A. Lortie, «Grandes voiries : permanence des tracés et fluctuation des écritures », in

C. Prélorenzo (dir.), Infrastructures, villes et territoire, Paris, L'Harmattan, 2000,

p. $155-160$.

11. C. Brown (dir.), Histoire générale du Canada, Montréal, Boréal, 1987, ainsi que P.-

A. Linteau, Histoire de Montréal depuis la Confédération, Montréal, Boréal, 1992, p. 440 sq.

12. G. Bessette, La bagarre, Montréal, Cercle du livre de France, 1958.

13. Le projet pour le Centre Eaton est lancé par la filiale canadienne du même groupe américain, Webb \& Knapp, qui a réalisé la Place Ville-Marie.

14. H. Aubin, Les vrais propriétaires de Montréal, Montréal, Éditions l'Étincelle, 1977, p. 70.

15. Housing and Urban Growth in Canada, Ottawa, Central Mortage and Housing corporation, 1956 (traduction de l'auteur).

16. Ibid.

17. Société de développement de Candiac, titre d'un dépliant publicitaire présentant des maisons types, sans date, vers 1960.

18. Étude menée pour le compte d'I. Rudberg, le promoteur immobilier de l'immeuble CIL, conçu par l'agence américaine SOM.

19. «À travers un heureux mélange de prévoyance éclairée, d'initiative privée et de chance, Montréal est sur le point de devenir la première ville du $\mathrm{xx}^{\mathrm{e}}$ siècle en Amérique du Nord. Et ce qui fait de ce centre une exception n'est pas tant ses tours que la ramification de leurs racines en un réseau à niveaux multiples de commerces, de systèmes de transport et de commerces. »P. Blake, « Downtown in 3D », The Architectural Forum, vol. CXXV, n 2, sept. 1966, p. 33.

20. R. Banham, Megastructure, Urban Future of the Recent Past, New York, Harper and Row, 1976. 
21. H. Aquin, L'invention de la mort, édition critique établie par M. Dumais avec la collaboration de C. Lamy, Montréal, Bibliothèque Québécoise, 2001.

22. J. Cimon, « Montréal innombrable », Liberté, n 28, vol. V, n 4, 1963, p. 371.

23. Voir à ce sujet les travaux d'A. Wallace, de l'Université de New York.

24. P. Gravel, À perte de temps, Montréal, Toronto, Parti Pris, House of Anansi Press, 1969 , p. 26.

25. H. Aquin, «Essai crucimorphe ", Liberté, $\mathrm{n}^{\circ} 28$, vol. V, n 4, 1963, p. 323-325.

26. « Ha ! Henri-Julien, Drolet, voilà l'authentique... Ce sont des îlots étroits, aux façades parallèles, dont l'arrière donne sur une venelle partagée. Chaque îlot allongé est structuré par cette ruelle et sa vie collective grouillante. Et vous y trouverez parfois un étroit carré d'herbe pelée encombré de dix adultes et de Dieu sait combien de gamins hurlant » (traduction de l'auteur). H. Hood, Around the Mountain, Scenes from Montreal Life, Toronto, Peter Martin Associates, 1967, p. 21.

27. M. Castells, Luttes urbaines et pouvoir politique, Paris, François Maspero, 1975.

28. R. Carrier, Le deux millième étage, Montréal, Éditions du Jour, 1973, p. 25.

29. Dénommé également «Golden square mille », compte tenu des fortunes familiales qui s'y trouvaient.

\section{RÉSUMÉS}

L'urbanisme et l'architecture des années 1960 ont souvent été décriés. Portés par un optimisme ravageur, il est vrai qu'ils ont laissé plus d'une ville éventrée, traversée de voies autoroutières, orpheline de quartiers populaires rénovés... À certains égards, Montréal n'échappe pas à la règle. $E t$, pourtant, si d'aucuns considèrent le legs de cette époque comme catastrophique, les mêmes s'accordent à lui reconnaître des qualités exceptionnelles, telles que son réseau sous-terrain de galeries commerciales, son métro, certains immeubles remarquables et la vitalité d'un centre ville où se côtoient actifs et résidents, classes sociales et cultures diverses. Et, force est de constater que les Montréalais s'identifient aujourd'hui autant à la ville populaire et historique qu'à celle, moderne, dont la structure et la forme ont été conçues dans les décennies après la seconde guerre mondiale. C'est à une interprétation de ce phénomène d'identification que cet article invite: quelle était la ville qui a été transformée? Quels artefacts nouveaux l'ont remplacée ? Quelle réceptivité ont-ils connu, à Montréal et ailleurs dans le monde ? Ce sont ces questions, parmi d'autres, qui sont abordées en texte et en image.

André Lortie, Montreal 1960, the motivations for reidentification

The urban planning and architecture of the 1960s have often been disparaged. It is true that they were driven by an irresponsible optimism that left more than one city ravaged, tear open by expressways, orphan of its renovated popular districts. In some respects, Montreal was no exception to the rule. And yet, while some consider the legacy of the decade to be catastrophic, they still recognize that it had some outstanding qualities, such as Montreal's underground network of shopping centres, its metro, some remarkable buildings, and the vitality of a city core in which workers and residents from different social classes and cultures all rubbed shoulders. And one has to note that Montrealers today as easily identify themselves with the popular and historical city than with the modern one, which structure and form were conceived and achieved 
in the decades following World War II. This article is an attempt to interpret this identification phenomenon: which was this city that was deeply transformed? What new artefacts replaced it? What reception did they experience, in Montreal and abroad? These are the questions, among others, that are discussed, through texts and images.

INDEX

Mots-clés : photographie, paysage urbain, rénovation urbaine

Keywords : photography, urban landscape, Montréal, réidentification, urban renovation

\section{AUTEUR}

\section{ANDRÉ LORTIE}

Professeur, École nationale supérieure d'architecture de Normandie, 27 rue LucienFromage 76160-Darnétal, Membre du Laboratoire architecture, culture, société, $\mathrm{XIX}^{\mathrm{e}}-\mathrm{XX}^{\mathrm{e}}$ siècles, École nationale supérieure d'architecture Paris-Malaquais, CNRS, ArchitectureUrbanisme-Société (AUS) a.lortie@wanadoo.fr 